Documentation et bibliothèques

\title{
Un service de bibliothèque aux handicapés physiques et visuels
}

\section{Réal Messier}

Volume 21, numéro 2, juin 1975

URI : https://id.erudit.org/iderudit/1055502ar

DOI : https://doi.org/10.7202/1055502ar

Aller au sommaire du numéro

Éditeur(s)

Association pour l'avancement des sciences et des techniques de la documentation (ASTED)

\section{ISSN}

0315-2340 (imprimé)

2291-8949 (numérique)

Découvrir la revue

Citer cet article

Messier, R. (1975). Un service de bibliothèque aux handicapés physiques et visuels. Documentation et bibliothèques, 21(2), 101-103.

https://doi.org/10.7202/1055502ar

Tous droits réservés (C) Association pour l'avancement des sciences et des techniques de la documentation (ASTED), 1975
Ce document est protégé par la loi sur le droit d'auteur. L'utilisation des services d'Érudit (y compris la reproduction) est assujettie à sa politique d'utilisation que vous pouvez consulter en ligne.

https://apropos.erudit.org/fr/usagers/politique-dutilisation/ 


\section{chronique
de la recherche}

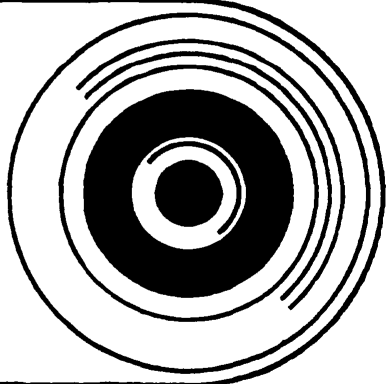

\section{Un service de bibliothèque aux handicapés physiques et visuels}

En février 1974, Guy Sylvestre, directeur général de la Bibliothèque nationale du Canada (BNC), annonçait la création d'un groupe de travail sur le service de bibliothèque aux handicapés physiques et visuels. Le mandat du groupe était de conseiller la Bibliothèque nationale sur la planification d'un programme coopératif national visant à fournir un service de bibliothèque bilingue adéquat aux handicapés physiques et visuels du Canada.

Le comité, présidé par lan Wees, coordonnateur des collections et services spéciaux de la BNC, est composé d'une douzaine de personnes dont deux de la Colombie, deux du Manitoba, cinq de I'Ontario dont trois de la BNC et deux du Québec. Quatre membres du comité sont eux-mêmes des handicapés physiques ou visuels.

Devant l'ampleur du mandat, quatre sous-groupes ont été formés devant s'attacher à des aspects particuliers du problème.

\section{Droit d'auteur}

Le premier sous-groupe, celui du droit d'auteur, avait pour mandat d'étudier le droit d'auteur, particulièrement en ce qui concerne les ouvrages publiés au Canada, et enquêter sur les pratiques courantes des bibliothèques et des producteurs en ce qui concerne le droit d'auteur. Ce même sous-groupe verra à formuler une ligne de conduite sur la reproduction, pour les handicapés, de publications faisant l'objet du droit d'auteur.

Certaines bibliothèques offrant déjà un service aux aveugles ont établi des rapports avec les éditeurs afin d'obtenir la permission de modifier le support de certains ouvrages, mais uniquement sur une base de "gentlemen agreement». Les éditeurs paraissent très conservateurs face à une telle transformation, même s'ils ont la certitude que ce matériel circulera entre les mains des personnes qui sont reconnues du point de vue légal, comme étant handicapées physiquement ou visuellement.

Comme la Chambre des Communes doit étudier la question du droit d'auteur (copyright) au cours de la prochaine session, il fallait miser juste et au bon moment.

\section{Normalisation}

Le second sous-groupe devait aborder la question des normes de production et de catalogage des documents sonores et de ceux en caractères spéciaux.

II existe actuellement un seul document de normalisation, le COMSTAC Report, préparé par la Commission on standards and accreditation of services for the blind. Cet ouvrage fournit tous les détails pertinents concernant l'organisation d'un tel service. Ce même organisme a préparé un fascicule Standards for production of Reading Materials for the Blind and Visually Handicapped qui sert de guide technique et administratif pour l'édition de matériel sonore ou en braille.

À cause de l'origine américaine du rapport et de certains détails techniques qui ne s'appliquent pas au contexte canadien, il faudra adapter les recommandations à notre cadre constitutionnel.

Les recommandations de ce groupe touchent également les différents formats 
(commerciaux ou non), la qualité du produit et le personnel employé pour traduire le document en d'autres formats.

Ce même sous-groupe doit recommander des normes de catalogage pour les documents sonores afin d'obtenir une uniformité maximale. II semble que le Non-book Materials de la CLA et les Règles de catalogage anglo-américaines soient déjà largement utilisés par les bibliothèques qui offrent actuellement le service.

\section{Inventaire}

Cet autre sous-groupe devait faire une étude exhaustive des services de bibliothèque offerts actuellement au Canada. Ainsi, un questionnaire a été préparé et expédié à toutes les bibliothèques universitaires du pays, aux principales bibliothèques publiques, aux bibliothèques de collège et aux organismes s'occupant des aveugles (ex.: Institut national canadien pour les aveugles) afin de connaître la quantité de matériel (braille, gros caractère, cassette, disque, ruban magnétique) dont ils disposent, le personnel et le budget, la bibliothèque, les différents services offerts aux handicapés physiques et visuels.

\section{Environ 500 questionnaires ont été} expédiés aux différents organismes et 200 bibliothèques $\mathrm{y}$ ont répondu; de ce nombre, plus de la moitié (127) affirment ne pas offrir un tel service, ne pas avoir de tels documents et même ne pas connaître ce sujet.

C'est la première fois qu'une telle enquête est faite au Canada. Un premier relevé indique que les bibliothèques publiques de la Colombie et de la région de Toronto accordent un excellent service, qu'un service semblable existe dans les Prairies, mais qu'il est inexistant au Québec et dans les Maritimes. Certaines bibliothèques universitaires, notamment la Crane Library à la University of British Columbia, offrent un tel service.

Les formats que l'on retrouve le plus souvent sont le braille ou les «large print" et il semble que les nouveaux moyens techniques (disques, cassettes, rubans magnétiques) ne font qu'apparaître dans les collections.

II n'y a que des organismes privés (Institut Nazareth et Louis Braille) qui s'occupent des services de bibliothèques aux handicapés physiques et visuels. Quelques aveugles de la région de la Mauricie, bénéficiant des programmes d'initiatives locales, ont mis sur pied un service pour les étudiants aveugles de la région.

Le troisième sous-groupe, en plus de colliger toutes les données sur l'acquisition, la production des documents, la nature de la clientèle, les budgets annuels de ces services, devait fournir également des informations sur les services déjà existants dans d'autres pays ainsi qu'une liste descriptive des sociétés commerciales qui publient du matériel destiné spécialement aux handicapés physiques et visuels.

\section{Recommandations}

Le dernier sous-groupe est chargé de faire des recommandations sur le nombre approximatif d'handicapés qui ne peuvent utiliser les documents traditionnels et ceux qui sont susceptibles de bénéficier d'un tel service, sur le montant approximatif des fonds nécessaires pour établir un service adéquat pour cette catégorie d'usagers.

II y a, certes, la définition légale de l'aveugle, mais cela n'inclut pas les vieillards qui ont une acuité visuelle inférieure à la normale, d'où la difficulté de quantifier la clientèle possible d'un tel service. Un rapport du ministère des Affaires sociales sur la Situation des handicapés visuels au Québec précise qu'il $y$ a actuellement près de 7,600 aveugles dans la province.

La Division for the Blind and Physically Handicapped de la Library of Congress estime qu'il y a 7.5 personnes sur 1,000 qui souffrent de troubles physiques ou visuels. On estime que le service offert à cette catégorie de lecteurs coûte 7 fois plus cher qu'un lecteur normal.

Ce même sous-groupe doit aussi délimiter les rôles de la Bibliothèque nationale du Canada, des services provinciaux de bibliothèques publiques, des ministères provinciaux de l'éducation, des organismes œuvrant auprès des aveugles et des bibliothèques publiques.

Le comité en est actuellement à l'étape de la rédaction des recommandations et le champ des juridictions semble difficile à délimiter, puisqu'il y a la BNC et l'Institut national canadien pour les aveugles (INCA) qui sont des organismes nationaux, des 
organisations différentes ou identiques au niveau provincial s'occupant des bibliothèques scolaires et publiques, les activités de ces organismes devant se compléter dans le respect de la souveraineté culturelle de chacune des provinces.

Le mandat précise que le comité doit établir les mécanismes de fonctionnement du service, allant du choix des documents qui seront transcrits sur ruban magnétique, en passant par les étapes techniques jusqu'à la distribution par les bibliothèques dans leur milieu respectif.

Pour l'instant, ceci ne concerne que les services des bibliothèques publiques. Parallèlement, les ministères de l'éducation des provinces ont créé un Interprovincial Task Force Committee on Educational Materials for the Handicapped qui se penche sur ce même problème, mais au niveau éducationnel. Certains membres du groupe de la BNC siègent à l'autre comité, ce qui assure des mécanismes de coordination. Le ministère de l'Éducation du Québec et son Service des moyens d'enseignement a été invité à participer aux assises du groupe national, mais n'y a jamais participé.

Le rapport final du Groupe de travail de la BNC sur le service de bibliothèque aux handicapés devrait être rendu public au début de l'été et le programme mis en opération vers la fin de 1976.

\section{Réal Messier}

Service des bibliothèques publiques Québec

Délégué de I'ASTED auprès du Groupe de travail de la Bibliothèque nationale du Canada

Grâce à CHECKPOINT des centaines de bibliothèques ont réduit les pertes de livres coûteuses

Le système de contrôle CHECKPOINT I est le premier dans ce domaine depuis 7 ans, et pour cause: il a réduit les pertes de livres à moins de $1 / 2$ p. 100 en moyenne.

\section{CHECKPOINT présente aux bibliothèques un système de contrôle nouveau et perfectionné, le MK II}

Pensez aux économies que vous pourrez réaliser! Seul le Système MK II peut vous assurer que les fausses alarmes ne se produiront pas.

Si vous comparez le Système MK II avec d'autres systèmes, vous trouverez trois raisons pour préférer CHECKPOINT:

1) un meilleur contrôle:

2) des coûts moindres à l'achat et à l'entretien;

3) une utilisation plus aisée.

Sans doute avez-vous déjà cherché un moyen de diminuer les pertes de livres. Voilà l'occasion!

Pour obtenir de plus amples renseignements, prière de remplir le coupon ci-dessous et de nous le faire parvenir à l'adresse indiquée

Si vous en exprimez le désir, une démonstration pourra être faite sans obligation de votre part.

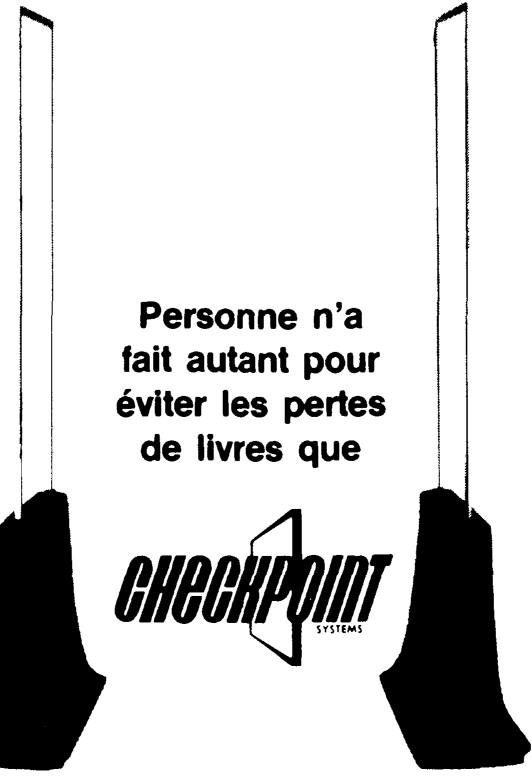

Veuillez me faire parvenir tous les renseignements sur le Système MK II.

CHECKPOINT SYSTEMS, INC.

Highway 17, East, Dept. DBI

Cumberland, Ontario

KOA 1S0, Canada

(613) 833-2203

Nom

Fonction

Bibliothèque

Adresse

Ville

Province

Indicatif régional

Filiale de

Logistics Industries Corp. 